July 1984

\title{
A Forty-Year-Old Woman with a History of Psychosis and a Seizure Disorder
}

\author{
Jeffrey R. Sarnoff, MD \\ Thomas Jefferson University Hospital \\ Robert E. Morrow, MD \\ Thomas Jefferson University Hospital \\ Sharon Riser, MD \\ Thomas Jefferson University Hospital \\ Ann E. Steel, MD \\ Thomas Jefferson University Hospital \\ Jacob Berman, MD \\ Thomas Jefferson University Hospital \\ Follow this and additional works at: https://jdc.jefferson.edu/jeffjpsychiatry \\ Part of the Psychiatry Commons \\ Let us know how access to this document benefits you
}

\author{
Recommended Citation \\ Sarnoff, MD, Jeffrey R.; Morrow, MD, Robert E.; Riser, MD, Sharon; Steel, MD, Ann E.; and Berman, MD, \\ Jacob (1984) "A Forty-Year-Old Woman with a History of Psychosis and a Seizure Disorder," Jefferson \\ Journal of Psychiatry. Vol. 2 : Iss. 2 , Article 6. \\ DOI: https://doi.org/10.29046/JJP.002.2.007 \\ Available at: https://jdc.jefferson.edu/jeffjpsychiatry/vol2/iss2/6
}

This Article is brought to you for free and open access by the Jefferson Digital Commons. The Jefferson Digital Commons is a service of Thomas Jefferson University's Center for Teaching and Learning (CTL). The Commons is a showcase for Jefferson books and journals, peer-reviewed scholarly publications, unique historical collections from the University archives, and teaching tools. The Jefferson Digital Commons allows researchers and interested readers anywhere in the world to learn about and keep up to date with Jefferson scholarship. This article has been accepted for inclusion in Jefferson Journal of Psychiatry by an authorized administrator of the Jefferson Digital Commons. For more information, please contact: JeffersonDigitalCommons@jefferson.edu. 


\title{
INTERDISCIPLINARY CASE CONFERENCE
}

In this issue of the Journal, we are introducing a new section, the Interdisciplinary Case Conference. Our goal will be to present psychiatric patients in whom pathology is demonstrable not only by interview, but also by physical examination, laboratory studies, and radiographic imaging techniques.

We are particularly grateful to Drs. Dubin, Bertoni, Streletz, and Ingalls for their participation in this endeavor, as well as for their kind words of encouragement.

\section{A FORTY-YEAR-OLD WOMAN WITH A HISTORY OF PSYCHOSIS AND A SEIZURE DISORDER}

\author{
JEFFREY R. SARNOFF, M.D.; ROBERT E. MORROW, M.D.; \\ SHARON RISER, M.D.; ANN E. STEEL, M.D.; \\ JACOB BERMAN, M.D.
}

Dr. Morrow, Resident in Psychiatry: The patient is a forty-year-old white female with a longstanding diagnosis of paranoid schizophrenia and temporal lobe epilepsy. She presented to the emergency room of this hospital following an overdose of phenobarbital and phenytoin (Dilantin ${ }^{\circledR}$ ). She was found to have a serum phenytoin level of 72 $\mathrm{mcg} / \mathrm{ml}$ (normal therapeutic range is $10-20 \mathrm{mcg} / \mathrm{ml}$ ) and a serum phenobarbital level of $52 \mathrm{mcg} / \mathrm{ml}$ (normal therapeutic range is $15-40 \mathrm{mcg} / \mathrm{ml}$ ). She stated that she took this overdose in an attempt to make up for missed dosages of these medications. Although she denied suicidal ideation at the time of this admission, she did admit to a history of voices telling her to drink bleach. After being admitted to the medical service and medically stabilized, the patient was transferred to the psychiatric service for further diagnostic evaluation and treatment.

This was the first Thomas Jefferson University Hospital admission for this patient, who was first hospitalized at the age of fifteen for an unspecified psychiatric disturbance. Over the past twenty-five years, she has been hospitalized numerous times for psychotic episodes that have been treated with ECT and neuroleptics. The patient reports a history of an unspecified type of seizure disorder that had been successfully controlled in the past with valproic acid, as well as a combination of medications that the patient could not recall. At the time of this admission, the patient was being followed for epilepsy at a different hospital. She was receiving phenytoin, $100 \mathrm{mgs}$ three times daily, and phenobarbital, $120 \mathrm{mgs}$ per day. She stated that prior to this admission she was having approximately one seizure every two weeks. In addition to phenytoin and phenobarbital, the patient had taken an unknown quantity of haloperi-

Drs. Sarnoff, Morrow, Riser, Steel, and Berman are first-year residents in Psychiatry. 
dol (Haldol ${ }^{\circledR}$ ) for the two months prior to admission. Her compliance with these medications and with psychiatric outpatient therapy has been erratic.

Other than a history of a seizure disorder, this patient's past medical history is remarkable for an appendectomy and for removal of sebaceous cysts. There is a past history of cigarette smoking not further quantified. The patient denied any history of alcohol abuse or abuse of illicit drugs. The family history is remarkable for unspecified psychiatric disorders in the patient's mother and maternal great uncle.

At admission to the psychiatric service, she was a thin, dishevelled white female who appeared her stated age. She was wearing one bedroom slipper. Her right arm and leg manifested a coarse resting tremor. Her speech was incoherent, often illogical, and frequently pressured and loud. Her affect was suspicious and hostile. The patient's thought content included paranoid delusions and a preoccupation with her seizure disorder and her auditory hallucinations. She denied suicidal or homicidal ideation. She was alert and oriented to person, place and time. No memory deficits were noted. Her ability to concentrate and abstract appropriately was impaired. Her insight and judgement were impaired.

The physical examination was remarkable for horizontal nystagmus, with the fast component to the left, decreased vibratory sensation in both legs, and mild ataxia. Hypertrichosis was noted. Pertinent laboratory examinations revealed a mild increase in her hemoglobin (14.2), hematocrit (43.2) and mean corpuscular volume (97). SMA-12, thyroid function studies, urinalysis, chest X-ray, and electrocardiogram were all normal.

The patient's hospital course may be described as follows. She was admitted to the psychiatric service on September 26th with elevated levels of phenobarbital and phenytoin. When the levels had decreased to within therapeutic range, these medications were restarted. Upon admission, the patient was also given Haldol ${ }^{\circledR}$, which was titrated slowly upwards in order to avoid precipitating seizures. Although the patient complained of repeated seizures, none were ever observed by staff. Throughout her hospitalization the patient remained psychotic, preoccupied with the idea that laser beams were controlling her, despite dosages of haloperidol to $80 \mathrm{mgs}$ per day. The patient frequently displayed angry outbursts. After a neurologic consultation was obtained, the patient was started on a course of carbamazepine (Tegretol $\left.{ }^{\circledR}\right)$. At that time, the patient's phenobarbital, phenytoin, and haloperidol were titrated downwards. However, the patient's psychosis worsened and her haloperidol was retitrated up to 80 mgs per day. There was marked difficulty in obtaining therapeutic serum levels of carbamazepine, which was thought to be a result of the induction of the microsomal enzyme system by phenobarbital and phenytoin. As the patient received increasing doses of carbamazepine, she began to exhibit prominent ataxia, intention tremors and a positive Romberg Sign. (At this time the patient arrived to be interviewed).

Dr. Dubin, Attending Psychiatrist: Could you tell me why you are in the hospital today?

Patient: I attempted suicide.

Dr. Dubin: What was going on?

Patient: Well, the Catholic church by way of what I call the laser wanted to give the 
Nobel peace prize I won to someone else. I said, “OK, give it to them.” We were having a slight argument as to who did what.

Dr. Dubin: Who were you arguing with?

Patient: In my own apartment?

Dr. Dubin: Yes.

Patient: You are not going to believe this. This was not hallucinations. And don't ask me where I went to medical school either. It's none of your business.

Dr. Dubin: Who were you arguing with?

Patient: The Catholic church. The precise person in the Catholic church? I don't know. All I know is the label, Zion Catholic Church. Therefore talk to me.

Dr. Dubin: He was there in the apartment with you?

Patient: Yeah, it was pretty wild.

Dr. Dubin: Has anything like this ever happened to you before?

Patient: Since 1969 it's happened.

Dr. Dubin: Before that you never heard voices?

Patient: No. I had no premonitions of hallucinations, audio or video.

Dr. Dubin: Do you have auditory hallucinations?

Patient: I'm very good at that.

Dr. Dubin: What kind of voices do you hear?

Patient: I hear voices on the inner drum. I hear your voice on the outer mechanism.

Dr. Dubin: What do the voices tell you?

Patient: Well, lately, they've been quite negative. They have told me to commit suicide. About a year ago I was sitting down and writing something down. Now what possible harm could that do?

Dr. Dubin: When you're out on the street, do you ever feel like people are talking about you?

Patient: No.

Dr. Dubin: Do you ever feel like people try to put thoughts in your head?

Patient: Indirectly, yes.

Dr. Dubin: How's that?

Patient: By constantly talking. So far I've been very lucky. I mean, if you're going to have grandeur, I mean, what the heck, why not have it all the way. I've talked to Lester Maddox of Georgia, and I've talked to the President of the United States. I called him nuts.

Dr. Dubin: Who is the President of the United States?

Patient: Don't you know? My argument is that it's Reagan and the Vice-President is George Bush.

Dr. Dubin: Do you ever feel that when you watch television there are special messages for you?

Patient: Well, not exactly just for me, because there is another writer involved. The reason is she's in fiction and I'm in non-fiction. No, it's the other way around. I'm fiction and she's non-fiction.

Dr. Dubin: Do you feel like you have any special powers?

Patient: They're talking to us, yes. 
Dr. Dubin: What kind of powers do you think you have?

Patient: I don't have any special powers. I was talking to you about the other sentence.

Dr. Dubin: You mentioned that in 1969 you started hearing voices. What happened then?

Patient: That was the year I was in jail.

Dr. Dubin: What were you in jail for?

Patient: The charge was child beating. I pleaded guilty. You can get the court transcripts free of charge. Just write to the court, that's all. I think the judge was a little prejudiced.

Dr. Dubin: You also have epilepsy. When did you receive that diagnosis?

Patient: In this hospital?

Dr. Dubin: No, in your life.

Patient: In 1956. The doctor said, quote, "I think your daughter has epilepsy."

Dr. Dubin: How old were you then?

Patient: Aw, come on. One of us has to subtract. Let's see. I'm forty now. Go on, take it from there.

Dr. Dubin: Why don't you help me?

Patient: You don't believe me either. Be my guest.

Dr. Dubin: So in 1956 you were told you had epilepsy. Did they start you on any medication?

Patient: No, I was already on medication from my appendix operation. The nut who did my appendix put me on phenobarb.

Dr. Dubin: What was happening that your mother took you to a neurologist?

Patient: Oh, a minor detail. I was having blackouts, at school and everywhere. My girlfriend said her mom thought I had epilepsy. So I said, "Wait just a minute." I immediately went to the library and told the librarian, I said, "What the heck's epilepsy? I can't even spell it." She said, "Go over there," and wrote it down. I looked it up. It said, "noncurable." That did it for me. Noncurable and living with my grandmother? Come on. Too much.

Dr. Dubin: That was in 1956 ?

Patient: Yes, then it was medically diagnosed.

Dr. Dubin: When did you first see a psychiatrist?

Patient: 1958.

Dr. Dubin: For what reason?

Patient: Suicide. My mother committed me.

Dr. Dubin: Were you hearing voices then?

Patient: No.

Dr. Dubin: Did you have feelings of grandeur then?

Patient: No.

Dr. Dubin: When did hearing voices and feelings of grandeur start?

Patient: No, I disagree with you. In 1969 they started.

Dr. Dubin: How many times have you attempted suicide?

Patient: I can't tell. I lost track.

Dr. Dubin: What usually leads up to your attempting suicide? 
Patient: Depression.

Dr. Dubin: When you get depressed, what are you like?

Patient: Stop operating.

Dr. Dubin: Do you lose weight?

Patient: I wish I could. No, I don't. Contrary.

Dr. Dubin: You mean, you start eating a lot?

Patient: Yes.

Dr. Dubin: Do you have problems with your sleep?

Patient: No, I use sleep as the great escape. Which it is. Sleep keeps me away from problems.

Dr. Dubin: When you get depressed, how long does it usually last?

Patient: Six months.

Dr. Dubin: Have you ever been treated for depression?

Patient: Yes, once, at a Jewish hospital. They gave me a needle in the rectum, in the rear end. For moods, mood swings. I'm trying to think of the medicine-.

Dr. Dubin: It wasn't lithium, was it?

Patient: Lithium carbonated.

Dr. Dubin: They treated you with lithium?

Patient: Yes. Every two weeks I went to them.

Dr. Dubin: Let me ask you one or two other questions. Do you still have seizures?

Patient: Yes.

Dr. Dubin: What happens when you have a seizure?

Patient : I don't know. I had a seizure yesterday. I don't know what happened. I was drinking coffee. The next thing I knew someone was helping me on the floor. How I got to the floor I don't know, but someone was helping me up.

Dr. Dubin: Do you ever get a warning —?

Patient: An aura?

Dr. Dubin: Yes.

Patient: No longer. I used to.

Dr. Dubin: What was that like?

Patient: A five second aura.

Dr. Dubin: What was that like?

Patient: That's the sixty-four thousand dollar question. I mean, those auras you just can't explain. I told them down at church very nicely, "Don't try and duplicate them."

Dr. Dubin: Do you ever have a period of a few days where you start to get more irritable and angry before you have a seizure?

Patient: No. I've thrown away the sequel.

Dr. Dubin: You've met Dr. Bertoni, the neurologist. Do you mind if he does a brief neurological exam?

Patient: No, I wanted to congratulate him on doing an excellent job. Go ahead. Be my guest.

Dr. Bertoni, Attending Neurologist: Could you please walk forward with one foot in front of the other.

Patient: I can't do it. 
Dr. Bertoni: Do the best you can. You have some difficulty doing this, don't you?

Patient: Yes, my duct system is off.

Dr. Bertoni: Could you please touch my finger, then touch your nose? Keep going back and forth. Now do the same thing with your left hand. Thank you. Now run the heel of your right foot up and down your left shin. Now change legs. There is minimal ataxia on finger-to-nose and heel-to-shin tests that is much more prominent on gait testing.

Patient: My duct system's off.

Dr. Bertoni: Please look directly at this light. Although nystagmus was commented on before, there is now none evident in the straight ahead primary gaze. At the time of initial exam her Dilantin ${ }^{\circledR}$ level was still quite high. Are you having any feelings of rotation?

Patient: No.

Dr. Bertoni: There is a small degree of nystagmus on downward gaze and extreme left gaze. Otherwise, the neurological exam was remarkable chiefly for decreased vibratory sensation in the lower extremities, and a tendency to fall backward on Romberg testing.

Patient: Now are you going to tell me what's wrong with my duct system?

Dr. Bertoni: Let me ask you another couple of questions first. Do you have the major kind of seizures or the other kind?

Patient: Yes, that's classified.

Dr. Bertoni: Have you ever had the kind of seizure where you bite your tongue and lose control of your bladder?

Patient: Oh, yes. The first one. Ask the authorities.

Dr. Bertoni: Do you ever smell odors in connection with these seizures?

Patient: No.

Dr. Bertoni: One thing that I would like to demonstrate before I stop is the patient's response to Romberg testing. Could you please stand with your feet together and close your eyes.

Patient: I can't stay like this. It interferes with my exercising.

Dr. Bertoni: Now extend your arms. Please touch your nose with your finger. Do that a couple of times. The patient is doing pretty well when given a distracting task to do. On other occasions she has tended to fall backwards.

Patient: I do better with my legs spread apart.

Dr. Dubin: Thank you, Dr. Bertoni. Are there any questions you'd like to ask us?

Patient: No, you're an angel.

Dr. Dubin: (The patient is escorted from the room.) There are several issues which may be discussed. First, does the patient have a partial complex seizure disorder? If so, what effect does this disorder have on her psychosis? There is a subgroup of patients with partial complex seizures who develop a schizophrenic-like psychosis. If this patient's history is correct, then she may be a part of this subgroup. Typically, this psychosis occurs approximately fifteen years after the onset of the seizure disorder. Another issue is how to treat this patient if she is indeed a part of this subgroup. It has been demonstrated that when one controls the seizure disorder, the psychosis worsens, and that when one controls the psychosis, the seizure disorder worsens. With this in mind, 
we must ask ourselves what would be the safest neuroleptic to use in the light of evidence that neuroleptics can lower the seizure threshold. First, I would like to ask Dr. Streletz from the TJUH Department of Neurology to discuss the EEG findings.

Dr. Streletz: Although the EEG may support the diagnosis of partial complex seizures, this diagnosis is primarily based on clinical findings. For this reason, I would like to ask Dr. Bertoni to speak about the clinical aspects of partial complex seizures before discussing the EEG findings.

Dr. Bertoni: The first question that I always have regards the nature of the patient's spells. Often, these patients are poor historians. The most important evidence often must come from eye-witness accounts. Although the patient may not remember the seizure, per se, he may remember having a sore tongue or wet pants. If such evidence can be obtained in the absence of substance abuse or withdrawal, then one may suspect the presence of a recurring grand mal seizure disorder. As far as partial complex seizures are concerned, history is equally important. We like to have evidence of some phenomenon that is of temporal lobe origin, for example, olfactory hallucinations and deja vu experiences. Once a complete history is obtained, an attempt is made to classify the seizure disorder, not simply for the neatness of classifying them, but because the prognosis and treatment may depend on this differentiation. We also want to know whether or not the patient was taking any drugs that might lower the seizure threshold. In addition, what were the conditions under which the seizure occurred? For example, sleep deprivation or drug therapy can lower the seizure threshold. Finally, is the patient compliant with the treatment regimen that has been established for his seizure disorder? Once all of this information has been obtained, we seek corroborative evidence in tests such as EEGs and CT scans.

Dr. Dubin: Dr. Bertoni, it has been my impression from the literature that violence is rarely a consequence of partial complex seizures. Would you agree with this?

Dr. Bertoni: My belief is that almost anything is possible in an epileptic seizure. However, goal-directed violent behavior is extremely rare and very difficult to document. What most often happens is that in the period following a seizure these patients are bombarded with stimuli from bystanders who may be trying to help, for example, by restraining them, and they subsequently become combative.

Dr. Streletz: I agree with Dr. Bertoni that violent behavior among epileptic patients is most commonly seen in the post-ictal period. I am reminded of Dr. Faulkner's study of temporal lobe epileptics who had a history of aggressive and violent episodes (1). Most of these patients were schizophrenic and were found to have organic pathology on histological exams, at the time of temporal lobectomy. That is to say, small A-V malformations, mesiotemporal sclerosis, low-grade gliomas, or hamartomas of the temporal lobe were found in the resected tissue. The behavioral abnormalities improved after resection. This should help us understand that temporal lobe epilepsy is not a disease in and of itself, but rather a symptom of underlying pathology. The EEG may be helpful in localizing the source of this pathology, but rarely the precise etiology.

Looking at this patient's EEG, we can see a right antero to mid temporal focus of sharp waves and spike activity associated with some slowing in that area. In this 
patient, it is best demonstrated during sleep, an event which often activates interictal epileptiform activity. The persistent slowing that is seen throughout this patient's recording, that is to say, a delta focus in association with the noted epileptiform activity, may suggest an underlying structural lesion. Otherwise, the epileptic spike is probably arising from disordered synaptic function in the temporal lobe, an event that is correlated with partial complex seizures.

Dr. Bertoni: How do you distinguish the changes you've noted from the effects of medication such as phenothiazines?

Dr. Streletz: Drug effects commonly cause general changes in the EEG without localization, generally in the beta frequency. When benzodiazepenes are used, one induces excess high-amplitude fast activity in the frontal regions. This patient's background activity is not disorganized; there is no evidence of excessive fast or slow activity. Her recording is essentially remarkable for the seizure focus described.

Dr. Dubin: What percentage of patients with partial complex seizures have EEGs that are actually within normal limits?

Dr. Streletz: With standard EEG electrode placement, approximately ten percent of these patients may have a normal EEG. With more specialized electrode placement, however, these patients can sometimes be shown to have a seizure focus in the temporal lobe. An example of this is the placement of electrodes in the nasopharyngeal region (approximately $20 \mathrm{~mm}$ from the temporal lobe). Video-EEG correlation may also be useful in helping to identify these patients, particularly patients who are thought to have psychiatric disorders, because their EEGs and behavior may be simultaneously monitored.

Dr. Dubin: May we examine the CT scan?

Dr. Bertoni: The CT scan was done after the patient had been in the hospital for approximately one month. We can see that there is a little more space around the cerebellum than is normally seen and the fourth ventricle may be slightly larger than normal, but looking at the temporal lobes there is no evidence of abnormality. On the contrast-enhanced study, no temporal lobe lesions are seen either. The extra space around the patient's cerebellar vermis, however, may correlate with her mild clinical feelings of midline ataxia. Finally, there is some calcification of the basal ganglia and choroid plexus noted that is not of clinical significance in this patient.

Dr. Dubin: Let us turn to a discussion of possible psychopharmacological approaches to this patient.

Dr. Ingalls, Clinical Psychopharmacologist: The treatment of patients with both seizure disorders and psychosis can be extremely difficult. There is relatively little literature on this subject because the number of studies on sufficiently large patient population is small. The most recent study is based on drug infusions of the guinea pig hippocampus to elicit seizure activity, using this as a model for human seizure activity. This study, published in the Archives of General Psychiatry (2), refutes an earlier study in $J A M A$ (3) where the epileptic side effects of various psychotropics were studied, with a rating given to each psychotropic based on their propensity to cause seizures. These ratings are based chiefly on literature review. While these findings may be controversial, it is known that all antipsychotics can lower the seizure threshold. 
This knowledge is of greatest value in the patient who had a known seizure disorder. These patients must always be adequately treated with anticonvulsant medication before psychotropics are begun.

Dr. Dubin: Are some antipsychotics safer than others?

Dr. Ingalls: General references (4) seem to suggest that high potency neuroleptics may be safer, but there are really no scientific studies to support that.

Dr. Dubin: Dr. Bertoni, one of the findings seen in this patient was consistent with cerebellar degeneration. How common is cerebellar degeneration in patients who are receiving Dilantin ${ }^{\circledR}$ ?

Dr. Bertoni: In my practice, I am careful to monitor tandem gait because once I start seeing problems with that test, especially if the serum level of Dilantin ${ }^{\circledR}$ is in the therapeutic range, I consider switching to another anticonvulsant medicine. Some cerebellar ataxia is frequently encountered in patients who have received Dilantin ${ }^{\circledR}$ over a long period of time.

Dr. Dubin: Which medicine would you switch the patient to?

Dr. Bertoni: In most cases, if there is an adequate history to support the diagnosis of temporal lobe epilepsy, the drug of choice may not be phenytoin, but carbamazepine.

Dr. Dubin: Are you convinced that this patient has partial complex seizures?

Dr. Bertoni: I must say, I always have doubts, especially in patients who provide very little reliable information. I think that our first responsibility is to do the least harm possible. Only after careful evaluation of the risk-benefit ratio should we proceed with a course of therapy. In this patient, we do have some history of tongue biting and loss of bladder control, as well as EEG evidence to support the diagnosis of a seizure disorder, the source of which is the temporal lobe.

Dr. Ingalls: Often the question arises in the acute psychotic patient who needs relatively large dosages of antipsychotics if there is any benefit from temporarily increasing their dosage of anticonvulsant medication to obtain supratherapeutic levels. Could you comment on this?

Dr. Bertoni: Well, I think if the level is within the normal range for therapeutic efficacy, you probably don't need any more, although this level may need to be near the high end of the range. There is some evidence in the older literature to suggest that supramaximal or toxic levels may in fact have an adverse effect. As an aside, here, I'd like to make a plea that when you measure serum levels of anticonvulsant medications, what you want to measure is the trough level, that is to say, the serum level preceding the next dose. Also, while the levels that we measure are typically the total (free plus bound) serum level of these drugs, special studies like free levels may be necessary in those patients whose protein binding has been altered. Finally, clinical response is always more important than the numbers.

Dr. Dubin: From a psychiatric standpoint, patients with partial complex seizures with concomitant psychosis can manifest almost every symptom commonly seen in schizophrenia. The most common presenting symptoms have been reported to be paranoia and auditory hallucinations. Possible clues to a differential diagnosis between primary schizophrenia and a schizophrenic-like psychosis that may result from partial complex seizures include the patient's degree of relatedness and any history of premorbid 
personality disorders, notably the social withdrawal and isolation that is commonly associated with schizophrenia.

This patient does not fit neatly into any of our established categories. My diagnosis for this patient would be schizoaffective disorder. As evidence for this, I would point out her history of suicide attempts, bizarre, disorganized thinking, grandiosity, and elated affect. My first suggestion regarding the treatment of her psychosis would be to obtain a haloperidol level, not because the significance of these levels is that well known, but to ascertain whether or not this patient is absorbing or metabolizing this drug at a rate that has rendered the serum level of this drug inadequate. If this level is indeed adequate and she remains psychotic, I could suggest a trial of lithium carbonate.

\section{REFERENCES}

1. Falconer MA: Reversability by temporal-lobe resection of the behavioral abnormalities of temporal-lobe epilepsy. NEJM 289: 451-455, 1973

2. Oliver AP, Luchins DJ, Wyatt RJ: Neuroleptic-induced seizures. Arch Gen Psychiatry 39: 206-209, 1982

3. Itil TM, Soldatos C: Epileptogenic side effects of psychotropic drugs. JAMA 244: 14601463,1980

4. Baldessarini RJ: The "neuroleptic" antipsychotic drugs part 2: Neurologic side effects. Postgrad Med 65: 123-128, 1979 